\title{
Can a 15-Hour (Overnight) Urinary Catecholamine Measure Substitute for a 24-Hour Measure? ${ }^{1}$
}

\author{
DENISE JANICKI-DEVERTS ${ }^{2}$ \\ Carnegie Mellon University \\ SHELDON COHEN \\ Carnegie Mellon University
}

\author{
KELLY ZILLES \\ University of Pittsburgh \\ ANDREW BAUM \\ Departments of Psychology and Psychiatry \\ University of Pittsburgh
}

\begin{abstract}
This study examined whether $15-\mathrm{hr}$ and $24-\mathrm{hr}$ urinary catecholamine measures show comparable associations with other physiological measures that are expected to correlate with sympathetic nervous system activity. Participants (193 healthy adults) provided 24 $\mathrm{hr}$ urine samples that were collected in a controlled environment (hotel), and divided into 9-hr daytime and 15-hr overnight collections. On the same day, resting blood pressure (BP) was measured, and 8 samples of salivary cortisol were collected. Catecholamine (15-hr and 24-hr) measures were correlated substantially in the entire sample, and when examined separately by sex and by race. Both $15-\mathrm{hr}$ and $24-\mathrm{hr}$ epinephrine (E) correlated significantly with systolic BP and cortisol; 15-hr and 24-hr norepinephrine (NE) correlated significantly with cortisol. Correlation coefficients for 15-hr measures were similar, but not equivalent to those for 24-hr measures. Urinary catecholamines obtained via $15-$ $\mathrm{hr}$ overnight collection approximated but were not equivalent to catecholamines obtained via 24-hr collection. Overnight collection was associated with reduced power to detect significant associations of catecholamines with criterion variables, such that use of $15-\mathrm{hr}$ rather than 24-hr sampling required a relative increase in sample size of 1.32 times for $\mathrm{E}$ and 1.18 times for NE to detect similar effects. Researchers should weigh the costs of additional subjects to the benefit of decreased burden when choosing between the two sampling methods.
\end{abstract}

Urinary catecholamine excretion is a marker of sympathetic nervous system (SNS) activity that frequently is used by psychologists as a physiological measure of chronic stress. Measurement of epinephrine (E) and norepinephrine (NE) in urine, rather than plasma, is considered superior in this regard, as urinary levels provide an integrated representation of catecholamine secretion during an extended period (Weinkove, 1991).

${ }^{1}$ This research was supported by grants to the Pittsburgh Mind-Body Center from the National Heart Lung and Blood Institute (HL65111 and HL65112) and by the John D. and Catherine T. MacArthur Foundation Network on Socioeconomic Status and Health. The authors thank the study participants and staff, and Ellen Conser for her comments on an earlier draft of the manuscript.

${ }^{2}$ Correspondence concerning this article should be addressed to Denise Janicki-Deverts, Department of Psychology, Carnegie Mellon University, 5000 Forbes Avenue, Pittsburgh, PA 15213. 
A preferred strategy for the sampling of urinary catecholamines has been 24 hr urine collection, as it permits estimation of global sympathetic activity over the course of an entire day (Steptoe, 1987). An important problem associated with 24-hr sampling is that it creates a substantial burden for participants. Daytime urine collection is invasive. Participants must carry containers with them and find appropriate storage for their collected voids throughout the length of an entire day. Given these difficulties, adherence with 24-hr protocols tends to be poor for maintaining participation and measurement accuracy.

An alternative and less burdensome approach is $15-\mathrm{hr}$ overnight urine collection. Participants begin sampling in the early evening (e.g., 5:00 or 6:00 p.m.) and continue to collect urine from all voids until 8:00 or 9:00 a.m. the following day. The comparability of overnight and 24-hr sampling has been debated. Mainly, it is argued that overnight measures miss the behaviorally active part of the day. The present study evaluates the comparability of 15-hr and 24-hr samples in a study of risk factors for viral infection.

The main argument against the use of overnight collections is that the shorter sampling period may be less representative of typical catecholamine excretion rates than is collection during a full 24 hours. White, Brunner, and Barron (1995) addressed this issue in a small sample of university employees $(n=24 ; 71 \%$ female). Comparison of 24-hr and 8-hr overnight samples revealed large correlations $(r=.54-.81)$ between catecholamine measurements derived from each of these two methods (White et al., 1995). The relative increase in sample size required by employing overnight collection to achieve the power associated with a 24-hr protocol was modest.

The current study extends the work of White et al. (1995) in several ways. First, we use an overnight sampling period of 15 hours, rather than 8 hours. The 15-hr overnight sampling increases the representativeness of the measure without substantially influencing the invasiveness of the collection procedure. Also, we determine if White et al.'s findings will generalize to a large, diverse (51\% male; $44 \%$ non-White) sample, and we examine the associations separately by gender and race/ethnicity. Finally, we examine whether effect sizes differ when correlating the $15-\mathrm{hr}$ and $24-\mathrm{hr}$ samples with other physiological markers.

\section{Method}

\section{Participants}

Data were collected between the years 2000 and 2004. Participants were 193 individuals ( 95 male, 98 female) aged 21 to 55 years $(M$ age $=37.3, S D=8.8$ ) who responded to newspaper advertisements soliciting participants for studies of psychosocial risk factors for upper respiratory infections. Of the 193 participants, 108 (56\%) were White, 72 (37\%) were African American, and 13 (7\%) indicated other 
racial/ethnic categories. The median income was $\$ 17,500$ (range $\$ 2,500$ to $\$ 162,500)$ and mean education was 13.76 years $(S D=2.21)$. Nearly half of the sample $(47.2 \%)$ were smokers and the mean body mass index (BMI) was 28.98 ( $S D=$ 7.08). Here, we report analyses of baseline data obtained prior to any of the parent study-related interventions. All components of the study received IRB approval and subjects were paid $\$ 820$ for completing all aspects of the parent study.

Potential study participants were screened by telephone and later interviewed by a physician. Exclusion criteria included pregnancy or current breastfeeding, diagnosis of chronic illness or psychiatric disorder, or history of nasal surgery. Individuals also were excluded from participation if they were regularly taking medication, with the exception of the following medications: birth control, hormone replacement therapy, analgesics, and topical eczema/psoriasis medications.

\section{Catecholamines}

Urine collection. Urine collection took place under controlled conditions during a $24-\mathrm{hr}$ period of quarantine in a hotel. Participants were provided with 1-liter specimen bottles containing a preservative (sodium metabisulfate), and were instructed to collect all urine voids during the target period, which was divided into two stages. Stage 1 began at 5:00 p.m. and ended at 8:00 a.m. the following morning. Urines collected during Stage 1 comprised the overnight or 15-hr sample. Stage 2 began at 8:00 a.m. and ended at 5:00 p.m. Participants were instructed to save overnight samples and the first morning void in one container, and voids collected during Stage 2 in a second container. All samples were stirred thoroughly, separated into $7 \mathrm{ml}$ aliquots, and stored at $-20^{\circ} \mathrm{C}$ until later analysis. Concentrations of epinephrine (E) and norepinephrine (NE) were derived from the 9-hr and 15-hr samples separately, and these values were combined by multiplying concentration by sample volume, averaging the resulting values, and dividing by the total volume, yielding a weighted average concentration. There were 5 participants who failed to provide Stage $1(15-\mathrm{hr})$ urine samples, and 1 participant was missing the Stage 2 (9-hr) sample. Thus, $15-\mathrm{hr}$ data were available for 188 participants, and 24-hr data were available for 187 participants.

Sample preparation. Samples were thawed and centrifuged prior to analysis by solid phase extraction (SPE) and high performance liquid chromatography (HPLC). In preparation for SPE, 3-ml aliquots of urine in duplicate, $3 \mathrm{ml}$ of a standard solution (Bio-Rad) and $3 \mathrm{ml}$ of a normal quantitative urine control (Bio$\mathrm{Rad}$ ) each were mixed with $50 \mu \mathrm{l}$ of internal standard (DHBA, Bio-Rad), $5 \mathrm{ml}$ of dilution reagent (30 mM ammonium acetate, $2.7 \mathrm{mM}$ EDTA; $\mathrm{pH} 7.5)$ and $100 \mu \mathrm{l}$ of $0.5 \mathrm{M} \mathrm{NaOH}$. The $\mathrm{pH}$ of each mixture was adjusted to $6.5 \pm 0.5$ by adding 0.5 $\mathrm{M} \mathrm{NaOH}$. The solutions were absorbed onto a SPE column filled with Bio-Rex 70 cation exchange resin (50-100 mesh) and allowed to drain. The columns were washed with dilution reagent and distilled water and eluted with $6 \mathrm{ml}$ of $3.6 \mathrm{mM}$ 
ammonium pentaborate. The eluate was collected and injected into the high-performance liquid chromatograph.

Liquid chromatography. HPLC was performed with a Beckman Ultrasphere $\mathrm{C}_{18}$ ODS $5 \mu \mathrm{m}$ column $(4.6 \times 15 \mathrm{~cm})$. The system included a pump (ESA Model 583), an autosampler (ESA Model 542), and an electrochemical detector (ESA Model 5200A). The mobile phase consisted of $50 \mathrm{mM} \mathrm{NaH}{ }_{2} \mathrm{PO}_{4}, 33.6 \mu \mathrm{M}$ EDTA, $0.43 \mathrm{mM}$ SOS, and a $\mathrm{pH}$ of 3.5. The $\mathrm{MeOH}$ ratio was 95:5, and the flow was set at $1.2 \mathrm{ml} / \mathrm{min}$. Peak heights for $\mathrm{E}, \mathrm{NE}$, and the internal standard were measured with ESA 501 software.

\section{Cortisol}

Salivary cortisol samples were obtained on the same day in the hotel as were the 15-hr and 24-hr urines. There were eight saliva samples collected throughout the day: immediately at wakeup (approximately 7:15 a.m.), $1 \mathrm{hr}$ after wakeup, at 10:00 a.m., at 11:55 a.m., at 1:00 p.m., at 3:00 p.m., at 5:00 p.m., and at 10:00 p.m. To provide a sample, participants placed a roll of cotton into their mouths and chewed on it for approximately $30 \mathrm{~s}$ or until the roll became saturated. Once saturated, the cotton roll was placed into a labeled collection tube or salivette (Sarstedt, Rommelsdorft, Germany) on which participants wrote the time that the sample was taken. Compliance was enhanced by an alarm watch that sounded at each sampling time, and presented a unique code that the participant wrote on the collection tube. Salivettes were stored at $-20^{\circ} \mathrm{C}$ until later analysis.

Cortisol level was determined by ELISA-based competitive binding assay procedures (Salimetrics, State College, PA). The intra-assay coefficient of variation ranged from $3.9 \%$ to $7.1 \%$. Interassay variability was less than $7 \%$. The daily cortisol value was corrected (residualized score) for wakeup time on the day of measurement. Area under the curve (AUC) was then calculated for each day to measure total free-cortisol release.

Cortisol samples were included in analyses for a targeted collection time if they were taken within a designated window surrounding that time $( \pm 0.5 \mathrm{hr}$ for the first sample and $\pm 1.0 \mathrm{hr}$ for the remaining samples). AUC measures were computed only for those who had data for the first three samples (where decline in the rhythm is steeper) and at least two of the remaining four samples (when the rhythm is flatter). There were 7 participants who were excluded for not meeting these criteria.

\section{Blood Pressure}

Resting systolic blood pressure (SBP) and diastolic blood pressure (DBP) were measured while participants were in the hotel between 8:00 a.m. and noon on the morning prior to the start of urine collection. While they were in a seated position, participants were asked to remain still for a period of $10 \mathrm{~min}$, after 
Table 1

Correlation Coefficients of 15-Hr With 24-Hr Catecholamine Excretion

\begin{tabular}{lccccc}
\hline & $\begin{array}{c}\text { Sample } \\
(n=187)\end{array}$ & $\begin{array}{c}\text { Male } \\
(n=91)\end{array}$ & $\begin{array}{c}\text { Female } \\
(n=96)\end{array}$ & $\begin{array}{c}\text { White } \\
(n=106)\end{array}$ & $\begin{array}{c}\text { African } \\
\text { American } \\
(n=69)\end{array}$ \\
\hline Epinephrine & $.87 * * *$ & $.86 * * *$ & $.90 * * *$ & $.94 * * *$ & $.78 * * *$ \\
Norepinephrine & $.92 * * *$ & $.92 * * *$ & $.92 * * *$ & $.91 * * *$ & $.92 * * *$ \\
\hline
\end{tabular}

$* * * p<.001$.

which a series of three BP measurements were taken. BP measurements were separated by $5 \mathrm{~min}$, during which participants were to remain seated with their arms resting on the table beside them. SBP and DBP were computed as the average of the three relevant BP measurements.

\section{Results}

\section{Correlations of 15-Hr and 24-Hr Urinary Catecholamine Measures}

Correlations of 15-hr and 24-hr catecholamine measures for the entire sample and separately by gender and race/ethnicity are displayed in Table 1. Because only a few participants endorsed a racial/ethnic identity other than White or African American $(n=13)$, race comparisons were confined to Whites and African Americans. As indicated by the table, 15-hr E and NE measures were highly correlated with corresponding 24-hr measures. Inspection of Table 1 further reveals that associations between $15-\mathrm{hr}$ and $24-\mathrm{hr}$ catecholamine measures were consistently strong both for men and women, and for Whites and African Americans.

Using the correlations reported for the entire sample, we calculated the relative loss in power to detect associations of urinary catecholamines with other variables of interest if 15-hr sampling were being used in place of $24-\mathrm{hr}$ collection. The relative efficiencies were as follows: $15-\mathrm{hr} \mathrm{E}=(.87)^{2}$ or $75.7 \% ; 15-\mathrm{hr} \mathrm{NE}=$ $(.92)^{2}$ or $84.6 \%$. Taking the inverse of each of these values, we estimate that use of 15-hr rather than 24-hr sampling would require a relative increase in sample size of 1.32 times for $\mathrm{E}$ and 1.18 times for NE to detect associations of interest.

\section{Correlations of Urinary Catecholamine Measures With Other Physiological Measures}

We then assessed whether E and NE values obtained via each of the two methods showed comparable associations with other physiological measures that 
Table 2

Correlations of Urinary Epinephrine and Norepinephrine With Blood Pressure and Salivary Cortisol

\begin{tabular}{|c|c|c|c|c|c|c|c|c|}
\hline & \multicolumn{4}{|c|}{ Epinephrine } & \multicolumn{4}{|c|}{ Norepinephrine } \\
\hline & \multicolumn{2}{|c|}{$24-\mathrm{hr}$} & \multicolumn{2}{|c|}{ 15-hr } & \multicolumn{2}{|c|}{ 24-hr } & \multicolumn{2}{|c|}{ 15-hr } \\
\hline & $r$ & $n$ & $r$ & $n$ & $r$ & $n$ & $r$ & $n$ \\
\hline Systolic BP & $.17^{*}$ & 187 & $.18^{*}$ & 188 & .11 & 187 & .10 & 188 \\
\hline Diastolic BP & .11 & 187 & .11 & 188 & .16 & 187 & $.14 \dagger$ & 188 \\
\hline Control AUC & $.32 * * *$ & 174 & $.25 * *$ & 175 & $.26^{* *}$ & 174 & $.22 * *$ & 175 \\
\hline
\end{tabular}

Note. $\mathrm{BP}=$ blood pressure. $\mathrm{AUC}=$ area under the curve.

$\dagger p<.10 . * p<.05 . * * p<.01 . * * * p<.001$.

are expected to correlate with SNS activity. Table 2 shows that both 15-hr and 24-hr hour urinary E correlated significantly with SBP and salivary cortisol, and both 15-hr and 24-hr urinary NE correlated significantly with cortisol. Comparison with $t$ tests of correlation coefficients associated with 15-hr and 24-hr measures reveals that associations of 15-hr E and NE with BP and cortisol did not differ significantly from correlations of 24-hr E and NE with these physiological measures.

Correlations of urinary catecholamines with BP and cortisol are presented separately by gender in Table 3. Among men, 15-hr but not 24-hr E was correlated significantly with SBP. The $t$-test comparisons reveal that the difference in size between the two correlations was not statistically significant. Among women, both 15-hr and 24-hr E and NE measures were correlated significantly with salivary cortisol. Correlations of cortisol with $15-\mathrm{hr}$ and $24-\mathrm{hr}$ catecholamine measures, respectively, were not significantly different.

Correlations of urinary catecholamines with BP and cortisol are presented separately for Whites and African Americans in Table 4. Among Whites, both 15-hr and 24-hr E and NE were correlated significantly with SBP, DBP, and salivary cortisol. Only the difference in size between the respective correlations of $15-\mathrm{hr}$ and 24-hr E with cortisol was statistically significant, $t(98)=2.49, p<.01$. Among African Americans, none of the urinary catecholamine measures were associated significantly with BP or cortisol. Correlation coefficients associated with 15-hr measures did not differ from those associated with 24-hr measures.

Given that the correlation between 15-hr E and SBP was significant only among the men in the sample - whereas the correlations between all catecholamine 


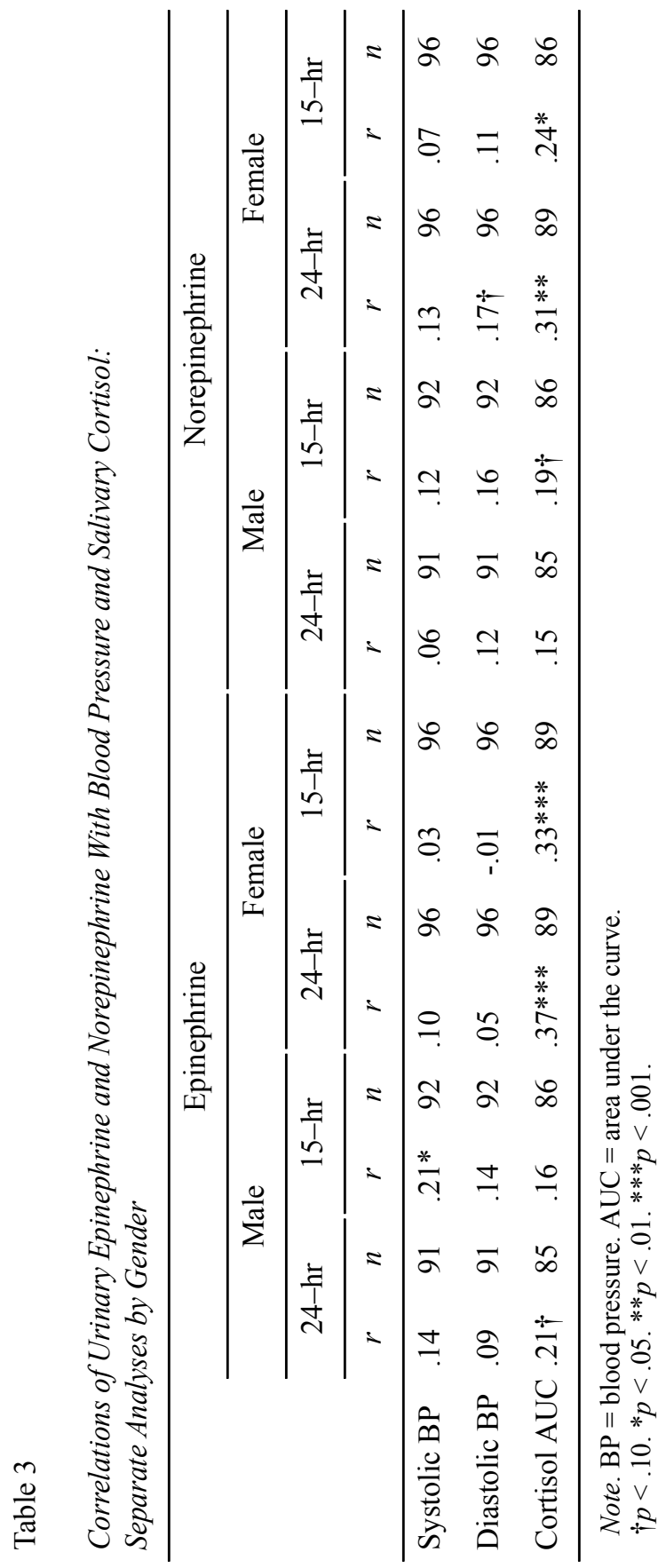




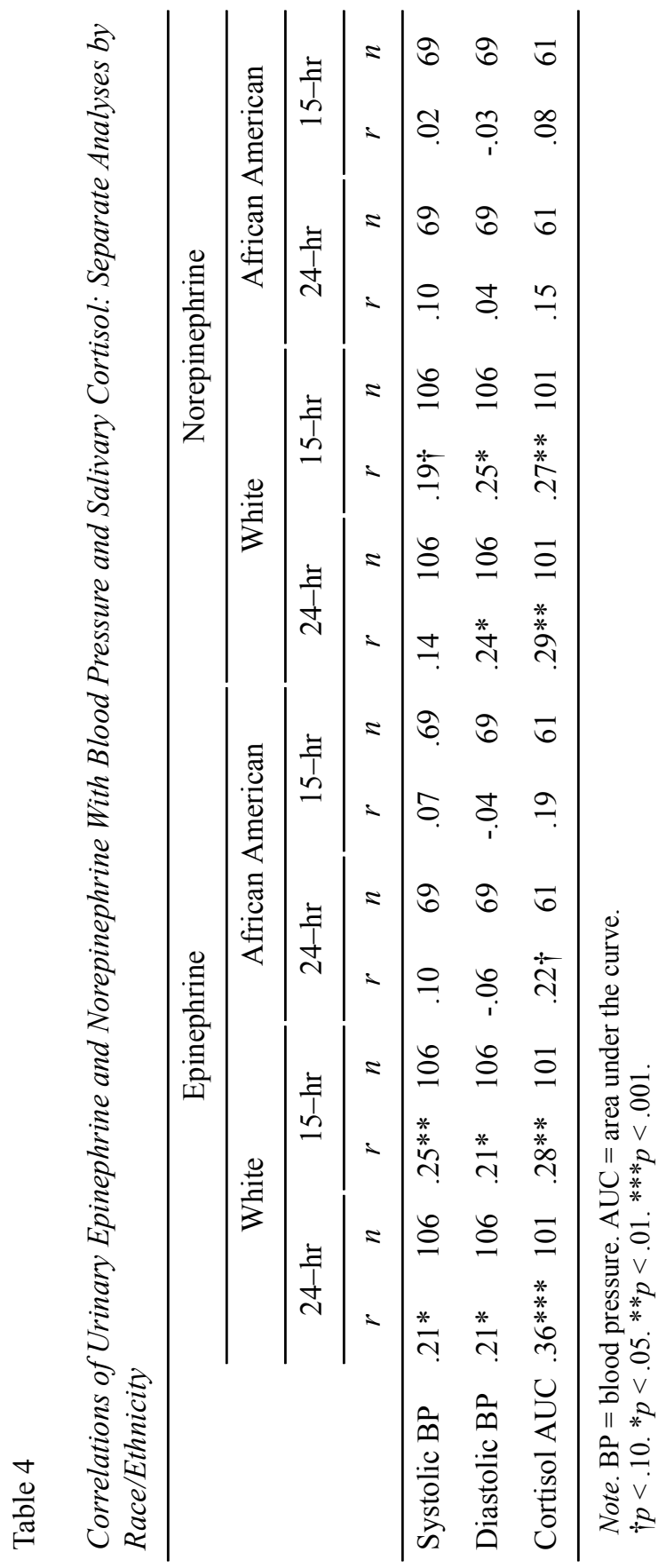


measures and cortisol were significant only among the women-we used $z$ tests to examine whether the size of the correlation coefficients differed between genders. Using similar comparison methods, we examined whether correlations of urinary catecholamines with SBP, DBP, and cortisol differed as a function of race/ethnicity. Results reveal that the correlation coefficients for each of the examined associations were similar across gender and race/ethnicity.

\section{Discussion}

Our data suggest that catecholamine measures obtained via 15-hr overnight urine collection are comparable to those obtained with 24-hr collection among a large, ethnically diverse sample of healthy men and women. Moreover, associations with other physiological markers that are expected to correlate with SNS activity were similarly comparable for $15-\mathrm{hr}$ and $24-\mathrm{hr}$ catecholamine measures.

Although White et al.'s (1995) findings and those reported here both suggest that catecholamine data obtained via overnight collection provide a reasonable substitute for 24-hr measures, the present associations between the two sampling methods are larger than those reported by White et al. There are two features of the present study that likely explain the greater similarity between overnight and 24-hr measures reported here. First, the overnight collection employed by the present study sampled catecholamine excretion over a standardized period of 15 hours, whereas the overnight collection in the previous study sampled urine over a period of approximately 8 hours. A second explanation for the larger correlations reported here is that the present sample was substantially larger than the sample in White et al.'s study. Individual differences that might have resulted in low correlations between overnight and 24-hr measures with their small sample would have been less influential here. Finally, participants in our study were quarantined during the collection period. Although they were allowed to move around the hotel floor (e.g., interact with others, watch television), the possibility of physical exertion was limited. This limitation may have contributed to a greater similarity between daytime and nighttime catecholamine levels than might be observed were measures not taken under controlled conditions.

A few additional limitations of the present study should be noted. The protocol of the parent study from which the present data were taken required participants to stay overnight in a hotel for 1 week. It is possible that individuals who are available to participate in this type of study may not be representative of the general population (e.g., participants may be more likely to be unemployed). Also, all female participants did not provide data during the same phase of the menstrual cycle. Cyclical fluctuations in ovarian hormones have been found to be associated with unchanged $\mathrm{BP}$ and heart rate, despite significant variations in plasma NE (Hirshoren et al., 2002). Thus, the lack of association between urinary catecholamines and BP among women may have been accounted for, to some 
extent, by menstrual-cycle effects. We explored this possibility by examining the association between urinary catecholamines and where female participants were in their menstrual cycle on the day of urine collection. Results showed no association between menstrual-cycle phase and catecholamines. Relatedly, it should be acknowledged that women taking oral contraceptives (OCs) or hormone replacement therapy (HRT) were not excluded from the present study. Research on the association between these two types of medication and catecholamine levels is limited, but estrogen replacement may be associated with lower levels of NE (Brownley et al., 2004). Thus, it is possible that the presence of women taking OCs or HRT may have influenced the results reported here.

In summary, the present results suggest that $15-\mathrm{hr}$ urinary catecholamine sampling may be an acceptable alternative to $24-\mathrm{hr}$ collection. The large correlations between overnight and 24-hr measures suggest that modest increases in sample size would be necessary to achieve similar power if collecting 15-hr rather than $24-\mathrm{hr}$ samples ( $32 \%$ for E; $18 \%$ for NE). Researchers will need to weigh the costs of additional subjects to the benefit of decreased burden when choosing between these two sampling strategies.

\section{References}

Brownley, K. A., Hinderliter, A. L., West, S. G., Grewen, K. M., Steege, J. F., Girdler, S. S., et al. (2004). Cardiovascular effects of 6 months of hormone replacement therapy versus placebo: Differences associated with years since menopause. American Journal of Obstetrics and Gynecology, 190, 10521058.

Hirshoren, N., Tzoran, I., Makrienko, I., Edoute, Y., Plawner, M. M., ItskovitzEldor, J., et al. (2002). Menstrual cycle effects on the neurohumoral and autonomic nervous systems regulating the cardiovascular system. Journal of Clinical Endocrinology and Metabolism, 87, 1569-1575.

Steptoe, A. (1987). The assessment of sympathetic nervous function in human stress research. Journal of Psychosomatic Research, 31, 141-152.

Weinkove, C. (1991). Measurement of catecholamines and their metabolites in urine. Journal of Clinical Pathology, 44, 269-275.

White, I. R., Brunner, E. J., \& Barron, J. L. (1995). A comparison of overnight and 24-hour collection to measure urinary catecholamines. Journal of Clinical Epidemiology, 48, 263-267. 